\title{
Multimedia information retrieval in the twenty-first century
}

\author{
Michael S. Lew
}

Published online: 21 March 2012

(C) The Author(s) 2012. This article is published with open access at Springerlink.com

Welcome, friends and colleagues. Just one decade in the past would show our field as being composed of a few scattered workshops. Since then there has been tremendous growth in both research and applications. In the past 4 years, as a community we have published over a 1,000 peer reviewed research papers in areas ranging from image and audio retrieval to scientific and medical image search. We have developed our own test sets, software libraries, evaluation benchmarks, workshops and major conferences (notably ICMR, CIVR, and MIR). Only one important piece was missing to make the picture complete: a research journal. Now, it is my pleasure to introduce the first journal focused on our field, The International Journal of Multimedia Information Retrieval (IJMIR).

Our new journal encompasses all topics related to retrieval, exploration, and mining of media databases and collections. It combines the vibrant areas related to retrieval of images, audio, video, and medical and scientific imagery with understanding of the human side of the system including the social aspects which are crucial to interactive search. An unarguable strength of our field is that it plays an important role in diverse scientific areas because many significant recent discoveries have been made through analyzing and searching local and international imaging databases.

In our journal, there are essentially two paper categories: regular papers (original research) and surveys. Regular papers are typically triple peer reviewed and are meant to represent the novel research from the community.

Surveys undergo a peer review from a senior community member and are meant to capture trends, illuminate critical problems, and give insight into the state-of-the-art. In

M. S. Lew (ه)

Leiden Institute of Advanced Computer Science,

Leiden University, Niels Bohrweg 1, 2333 CA Leiden, The Netherlands

e-mail: mlew@liacs.nl principle, they are frequently developed in discussions with editorial board members or by senior community members and will often have a representative from the journal as a co-author. In forthcoming planned issues, we will have surveys on topics ranging from interactive search to bio-medical image retrieval to social media search.

In this inaugural issue, it is our pleasure to present a survey on the important topic of feature selection in multimedia annotation titled, "The Heterogeneous Feature Selection with Structural Sparsity for Multimedia Annotation and Hashing: A Survey" by Fei Wu, Yahong Han, Xiang Liu, Jian Shao, Yueting Zhuang and Zhongfei Zhang (section editor). We are also honored to present extended versions of the best papers (top 2\%) from the ACM International Conference on Multimedia Retrieval (ICMR) and the ACM International Conference on Image and Video Retrieval (CIVR). The papers were invited (note that even invited papers undergo an editorial board review) based on the reviews of the program committee by the program chairs (ICMR: Alan Hanjalic, B.S. Manjunath and Shin'ichi Satoh; CIVR: Yannis Avrithis, Noel O'Connor, Daniel Gatica-Perez and Tat-Seng Chua). The best papers are "Large Scale Near Duplicate Image Retrieval by Kernel Density Estimation" by Wei Tong, Fengjie Li, Rong Jin and Anil Jain; and "Multimedia Semantics-Aware Query-Adaptive Hashing with Bits Reconfigurability" by Yadong Mu, Xiangyu Chen, Xianglong Liu, Tat-Seng Chua and Shuicheng Yan; "Multimodal Image Retrieval" by Stefan Romberg, Rainer Lienhart and Eva Horster; and "Bridging the Gap Between Expert and Novice Users for Video Search" by Martin Halvey and Joemon M. Jose.

Personally, I am proud of being a member of our research community. Collectively, we worked together to establish our community. We were the pioneers and we are the ones who are expanding the current frontiers. Moreover, recent citation 
impact indicators have shown that our contributions have been among the most influential in the field of multimedia and also in computer science.

We are living in an Age of Information, where multimedia information is being created and transferred worldwide in limitless quantities. Our role is to face the immense challenges of searching through this mountain of data and developing new paradigms which allow users to browse, interact, and share the information in intuitive ways. We are the ones who will make multimedia information accessible to the world of the twenty-first century.

Michael S. Lew

Editor-in-Chief

International Journal of Multimedia Information Retrieval

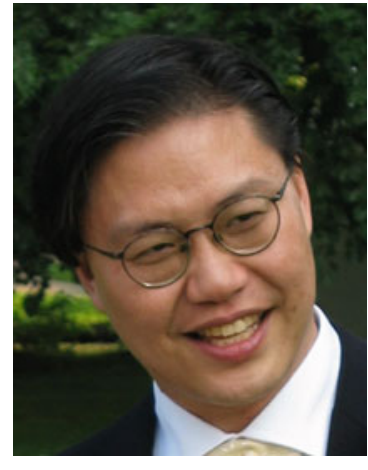

Open Access This article is distributed under the terms of the Creative Commons Attribution License which permits any use, distribution, and reproduction in any medium, provided the original author(s) and the source are credited. 